\title{
Automatic Sensation: Environmental Sensors in the Digital City
}

\section{Jennifer Gabrys}

\begin{abstract}
This paper discusses the use of environmental sensors, wireless networks and mobile media as technologies of sensation in the city. While these devices enable a "digital city," in many respects they appear to be immaterial, operating beyond sense. Further, drawing on two case studies presented by the Digital Cities project in Montreal, the paper considers how applications of environmental sensors and mobile media give rise to new conditions and questions for how we configure sense in the "digital city." The paper ultimately finds that sensors direct us toward new sites, assemblages and practices of sensation within the urban sensorium.
\end{abstract}

Keywords: environmental sensors, digital city, wireless networks, urban sensorium, locative sensation 


\section{Digital Cities, Digital Sense}

The "digital city," with its wireless networks, multimedia districts, virtual interfaces and mobile devices, is an environment that recasts orders of sensation in the city. In many respects, the digital city appears to be beyond sense. Digital operations are invisible, undetectable, virtual or an apparent simulation. With these seemingly imperceptible events, how do we locate a sense of the digital city, and how are we situated within it? Do we hear with our ears or with our mobile devices? Are we watching city scenes or are we viewing urban activity through our digital camera screens? Can sensor networks, as one of the more pervasive of these technologies, eventually realign our sensory taxonomies, so that sight, touch, smell, taste and sound are augmented and extended by registers of light, temperature, location and motion? The Digital Cities project based in Montreal investigates the terrain that specifically emerges at the intersection of environmental sensors and the multimedia city.1 This paper examines two urban installations carried out during the course of the project in order to explore issues that arise with automated sensation. The installations have included "Remote Sensors in the City," a network of "hotspots" with attached environmental sensors capable of reading temperature, humidity, light levels and proximity at short distances, and "Sampling the Park," a mediascape accessed through mobile technology which inscribes a city park in Montreal with sound- and image-based historical material that is triggered through GPS sensors. Based on these case studies, this paper considers how accurate and appropriate sensors are for describing our sensory experience of the city. The paper also questions whether attempts to describe and record the city through sensors render our first-hand encounters more or less palpable; and how entirely new patterns of sense may emerge through automated sensory networks.

This description of environmental sensors in urban environments is followed by a consideration of how sensors that gauge urban phenomena can relocate the sites of sensation. Perhaps a commonsense view of sense in the city would suggest that sensation occurs among urban inhabitants and their environment, that sensing contends with the mediating boundaries or membranes across which sensation is communicated. Yet the phenomena that sensors detect and transmit, from light levels to noise, are neither exclusive to nor even have their origin in the human body. Within the city of sensors, much information about sense is transmitted among machines, processed in tiny operating systems beyond the register of human detection. In this way, sensors suggest orders of sensation that extend beyond the body to an urban sensorium. In moving this discussion of sense beyond a register that is exclusive to the body, I hope to account for the role of technology in the formation of sense and to consider how the city constitutes a site and technology of collective sense. 2 Sensing is a process of configuring, of configuring into perception. Technologies enable this configuration. So, too, does the city perform as a technology, assembling and enabling orders of sensory experience. 


\section{Machines Making Sense}

The relationship between sensation and technology is long-standing. Articulating the ways in which this use and modification of sensation is distinctly human, Steven Connor writes that "while animals merely employ their senses, human beings construct theirs, as and through artifacts, or construct themselves through them" (Connor 2005). These artifacts are increasingly those instruments and technologies of sense through which sense is "enhanced and extended," including early examples of "telescopes, microscopes, microphones, loudspeakers and other such devices." Connor suggests that it is not just that these devices become mechanisms through which we sense, but also that they appear to take on sensations and sensing capabilities of their own. In other words, they appear to be "autonomized." He elaborates, "Thus the camera seems to 'see' for itself, and the phonograph to 'hear' on its own account, independent of a sensing subject" (Connor 2005). Environmental sensors in the current context do more than appear to sense independently, however; they are programmed (within limits) to sense independently, to adapt to changing circumstances and, effectively, to operate as "intelligent" sensing technologies. Sensation, in this case, appears to be displaced to technologies that operate as automatic and even "inhuman" organs of sense.

While sensors have existed for several decades, and were initially introduced in a military context during World War II, it is only recently that sensors have emerged as pervasive technologies. These devices, which currently range in size from a small book to a matchbox (but with aspirations toward the size of insects and dust), are calibrated to sense environmental phenomena including light, temperature, humidity, traffic, noise, air pollution, location, motion, traffic and more.3 Sensor networks, as conglomerations of minute sensors, may include swarms that number in the hundreds and thousands of devices. These clusters of sensors are situated to detect minute changes in the environment, communicating and processing this information locally and transmitting significant data for human detection. The strategy of these sensors is to scan the environment, to collect and process data, and only when necessity dictates to communicate this data. In this respect, these embedded devices or sensors have "three basic functions," including "detecting, computing and communicating context" (Beigl 2005: 52-61 at 59). Much of the sensorial processing that takes place, in this regard, occurs at a local level, among machines. This is part of the automatic, "smart" functioning of sensors; their primary operation is for machines to speak to machines, and not to people. In this way, sensors operate as autonomous, "self-configuring networks." They extend our senses, at once enabling a greater degree of data collection, but also allowing us to ignore that data until it registers a significant value. The intelligence of sensors, in this respect, has to do with the ability to know which sensory data is most relevant, and to communicate only that sensory information. 4 In order to communicate this relevant information, moreover, sensors rely on wireless networks that enable communication among machines and beyond to the Internet and satellites. A network of "hotspots" transmitting signals for wireless 
devices from sensors to laptops, for instance, will enable sensors to collect data and communicate information for display online.

The city and sites of transmission that were previously assumed to be "virtual" acquire a new level of permeability and influence with sensors. The operation of these sensors - automated and in the background - relocates the site and character of sensation in the city. Sensors are meant to sense beyond sense, to augment and detect invisible registers well out of range of human sensation. They may even, as Katherine Hayles writes, evolve to detect stimuli, and in this sense, "evolving new sensors implies constructing new worlds" (Hayles 2005: 139). Yet what do these delineations and detections of sense enable, and what do they exclude, particularly when we consider urban environments? Many sensor applications are deployed for the collection of data, for the measurement of phenomena and for the regulation of functions from traffic flow to lighting. Sensation is then bound to function, it is the trigger for other behaviors that will monitor and manage urban space. By managing urban environments, sensors effectively make urban environments. They contribute to the experience and sense of bodies and the city, from registers of speed, flows of traffic, to the expectant timing of transit networks. Sensors locate us, in this respect, in a new field of sensation - automatic, but also collective, and "out there."

\section{Relocating Sense in the City}

Environmental sensors detect phenomena that are quite distinct from those usually delineated as apprehensible by the five human senses. The five senses, which have also at various times and through different perspectives ranged from twelve to six to three, 5 are senses that emerge at the interface of the body and the environment. The delineation of these senses shifts in accordance with the assumed permeability of that interface, and even with its modification through various technologies and cultures. When Jonathan Crary discusses the emergence of an observing subject in the nineteenth century, he draws on a wider field of effects, from "discursive, social, technological, and institutional relations," to demonstrate how observers - perceiving subjects - are located specifically within and constituted through these relations (Crary 1990: 6). While he suggests that the observing subject emerges only through these relations, he at the same time demonstrates how the formation of a sensing body itself becomes a technology. Dispensing with an essential notion of the body and how we may arrive at its absolute conditions of sense, Crary builds up an account of how the perceiving body - primarily vision for the purposes of his argument - developed the capacity to sense in ways that were continuous with technological, economic and social developments at the time. Indicating the sort of "assemblage" through which the observer emerges, Crary draws on the writings of Walter Benjamin, in which we encounter an ambulatory observer shaped by a convergence of new urban spaces, technologies and new economic and symbolic functions of images and products - forms of artificial lighting, new use of mirrors, glass and steel architecture, railroads, museums, 
gardens, photography, fashion, crowds. Perception for Benjamin was acutely temporal and kinetic; he makes clear how modernity subverts even the possibility of a contemplative beholder. There is never a pure access to a single object; vision is always multiple, adjacent to and overlapping with other objects, desires and vectors. Even the congealed space of the museum cannot transcend a world where everything is in circulation (Crary 1990: 19-20). Sensation emerges here through an urban "convergence" - of technology, social fields and the sensing subject. But what's more, for this convergence to come together, the observer who passes through these spaces and fields must be "ambulatory," in continual motion, bound to the flux of modernity. In this scene, mobility and location emerge as enabling sensory registers as much as sight or touch. The observer senses the city - the location - by moving through it. Here, movement through the city and observation are a process of constant assemblage. The city itself seems to become a reciprocal sensing organism.

If we extend this logic further, how would this scene appear if we temporarily dislocated the observer as the principal processor of this array of stimuli? Would this same environment populated by automated sensors register different qualities of space? Would it appear as an entirely different city, where gardens perform selfphotography, mirrors communicate to products and architecture selfassesses its structural integrity? These questions point to the way in which the city, like the body as articulated by Crary, is a sensing technology as much as a delineated field of sensation. Within the city are spaces and sense technologies that mobilize and transform the possibility of sensation. Different cities emerge through these orders of sensation, in the same way that different observers emerge in relation to specific contexts. In this respect, sensors do not just replace one set of sensory divisions for another, but, rather, articulate and shift the field of sensory awareness. Sensors detect phenomena that do not exclusively register in planes of representation, that do not necessarily collapse into a point or surface of contact and that do not always mediate a boundary between inner and outer; but which, instead, perform new senses of position, situation, context and extension. The categories of sense that emerge and are made operable are direct responses to context, and are strategies for regulating that context. Sensors further suggest that location is one of the principal ways in which to assemble sensation. This relation between sensory technologies and the urban environment then effectively relocates - by extending - sense.

In this extended state, where "the nervous system is not contained within the body's limits," it becomes difficult to locate the edges of the body and environment. These "edges," as the editors of The Prosthetic Impulse note, shift on "both material and metaphorical" levels through the prosthesis (Smith and Morra 2005: 1-14). But where the interface between technology and body forms the basis for their prosthetic investigations, here I suggest that the murky edges between urban technologies and the body require equal attention. Sensors are not prostheses to the body, in the literal sense, but prostheses to the city (and the body secondarily). How do we construe these 
edges? Perhaps, as Susan Buck-Morss suggests, "the circuit from senseperception to motor response begins and ends in the world . . . As the source of stimuli and the arena for motor response, the external world must be included to complete the sensory circuit" (Buck-Morss 1992: 12). Reconfiguring inner and outer, we find that this division instead reveals a space of extension. In this sensory circuit, the boundary between inside and outside, virtual and actual, human and machine, is disturbed. Sensation occurs not in one or the other, but in the extension that occurs through these. In this space, we find our nervous systems "extended," as Marshall McLuhan has often suggested, into an environment composed of an "invisibilia of electric communications" - a space that constitutes the "fundamental materiality of contemporary social and cultural production" (Cavell 2002: 24; McLuhan 1964). What McLuhan describes is a nervous system, an electric media environment, which extends our senses and is an assemblage of sensing technologies. This sensorium, as an assemblage of sensing technologies, increasingly operates autonomously. Yet, for all these "autonomized" technologies, we still inhabit and move through this sensorium, we still operate as sensing bodies, but this too has changed.

\section{The Urban Sensorium}

Within this field, the Digital Cities project investigates ways of crafting sensation within the urban sensorium by employing sensors and designing location-based mediascapes. These sensing infrastructures span across multiple scales in the city, where a grid of automatic signals may give rise to place-specific applications. It is the movement within the wireless and sensorized city that enables shifting senses of the city, and reveals how the city is a technology that also senses us. The Digital Cities project, as part of its initial development, established a collaboration with the Montreal-based community group, Île sans fil (ISF), which resulted in the installation of an island-wide network of free wireless "hotspots." 6 These hotspots, located in public spaces, are primarily intended to allow free access to the Internet. But the presence of this relatively continuous wireless network not only enabled the use of mobile devices and allowed ready access to the Internet, it also facilitated the installation of environmental sensors that could detect various urban phenomena from temperature and humidity to light levels and proximity at short distances. Via wireless hotspots, these sensors then detect and transmit data, which is sent over the Internet to a server and stored in a database. While the Digital Cities sensor installations are not developed as extensive sensor networks, or swarms, they do perform many of the same sensing and data-collection functions. This infrastructure of wireless networks overlaid in public spaces then also becomes a sensing infrastructure. Public spaces transform into sites of intensified communication, increased surveillance and augmented sensation. While surveillance is enabled to a much greater degree by sensors, this important area of investigation is outside the scope of this paper. But all these "sites" of sensation appear to exist on the order of machines. How then do we locate and access this sensation afforded by real-time data? 


\section{The Urban Sensorium}

Within this field, the Digital Cities project investigates ways of crafting sensation within the urban sensorium by employing sensors and designing location-based mediascapes. These sensing infrastructures span across multiple scales in the city, where a grid of automatic signals may give rise to place-specific applications. It is the movement within the wireless and sensorized city that enables shifting senses of the city, and reveals how the city is a technology that also senses us. The Digital Cities project, as part of its initial development, established a collaboration with the Montreal-based community group, Île sans fil (ISF), which resulted in the installation of an island-wide network of free wireless "hotspots."6 These hotspots, located in public spaces, are primarily intended to allow free access to the Internet. But the presence of this relatively continuous wireless network not only enabled the use of mobile devices and allowed ready access to the Internet, it also facilitated the installation of environmental sensors that could detect various urban phenomena from temperature and humidity to light levels and proximity at short distances. Via wireless hotspots, these sensors then detect and transmit data, which is sent over the Internet to a server and stored in a database. While the Digital Cities sensor installations are not developed as extensive sensor networks, or swarms, they do perform many of the same sensing and data-collection functions. This infrastructure of wireless networks overlaid in public spaces then also becomes a sensing infrastructure. Public spaces transform into sites of intensified communication, increased surveillance and augmented sensation. While surveillance is enabled to a much greater degree by sensors, this important area of investigation is outside the scope of this paper. But all these "sites" of sensation appear to exist on the order of machines. How then do we locate and access this sensation afforded by real-time data?

\section{Sense as Synthesis}

A sensor detects and processes stimuli, and communicates this information typically through electrical signals. As such, sensors take up the role of processing stimuli in order to make perception. As Buck-Morss writes, "the powerfully prosthetic sense organs of technology are the new 'ego' of a transformed synaesthetic system. Now they provide the porous surface between inner and outer, both perceptual organ and mechanism of defense" (Buck-Morss 1992: 33). With sensor technologies, the synthesis that is sensation, the transformation of stimuli to perception, occurs in locations remote from our sense organs. With environmental sensors and mobile media, we access this sensation through interfaces that communicate processed sensation to us. This in turn has implications for how we interact with urban spaces, and for the sort of sensing technology that the city becomes. This situation gives rise to a possible sense of separation between environment and sense, between environment and media. The need to interpret and synthesize the vast array of sensations available becomes more pressing. As it turns out, the sought-after synthesis may occur most readily not through the mutation of our senses to align with media devices, but, rather, in the locations that we inhabit and the way that sensing 
technologies guide us in these spaces - beyond the interface and through the extended contours of the urban sensorium. In this, we arrive not at a delineation of discrete senses, but, instead, at the sites of sensation, which, as it turns out, are also the sites of synthesis. So while location-based sensors may trigger other events or memories or call upon an archive of environmental data, the locative triggering that occurs should arguably cause us to lift our eyes from the interface and direct us to the city that surrounds us. In this way, we can begin to see the city as a sensing technology - an urban sensorium that enables and registers orders of collective sense and synthesis.

This collective sensation is like the weather, a mood, or atmosphere that implicates us in and reminds us of the multiple locations and assemblages of sense.

\section{Sensing beyond Sense}

With the Digital Cities Project and the related Mobile Digital Commons Network, research is currently in process into the ways in which mobile gaming can become a way to design location-based experiences that direct our attention to places - from urban parks to forests and beyond. Similar projects, including "Sensor," a work by Carsten Nicolai shown in Potsdamer Platz, Berlin, during transmediale.06, bring the texture of sensor-based data to urban surfaces to reveal collective orders of sensation.7 This project in particular employs sensors to record and translate data related to traffic, light and noise in order to generate light signals and gradients over a building façade. These applications move environmental sensors beyond the function of regulating and recording and attempt to build interpretive architectures and location-based experiences. They at once reveal the located-ness and mediation of sense. They also engage in the ways in which location is an associative sense and gives rise to the interplay of the senses. Both Marshall McLuhan and David Howes discuss the "interplay of the senses" and "intersensorality," and here I suggest that locations - including the technologies of place - are critical to the formation of this interplay. In many respects, these projects seek to reveal the new sites of sensation that are made available by sensor-based, locative and mobile technologies. Connor discusses how through machines we "develop new kinds of perception." These new perceptive capacities, Connor suggests, perhaps in contrast to Buck-Morss, do not occur through prosthetic technologies that exclusively sever us from "our natural existence," but rather through technologies that allow us to develop new sensitivities that enable ongoing creativity, imaginative appropriation and improvisation - even by allowing us to "take leave of our senses" (Connor 2005). In taking leave of our senses, in moving beyond sense, we potentially arrive in new spaces of sensation. With the deployment of technologies that sense beyond sense, we may return to the convergences and locations that bring us to our senses and that locate us in a renewed urban sensorium. 


\section{Acknowledgements}

Michael Longford provided support and useful material in the preparation of this paper; Kathryn Yusoff provided valuable comments during the paper's development.

\section{Notes}

1. Information on the Digital Cities project and related Mobile Digital Commons Network can be found at http://www.digitalcitiesproject. net and http://www.mdcn.ca.

2. For a more thorough discussion of the role of technology in forming sensory environments, or an "economy of experience" see Hansen (2000). The work that Hansen draws on to develop this argument is Benjamin (1968: 217-52).

3. For a discussion on "smart dust" see Gabrys, Jennifer (forthcoming).

4. For more technical information on sensors, see Akyildiz, I.F., W. Su, Y. Sankarasubramaniam, E. Cayirci. (2002) and the UCLA Center for Embedded Networked Sensing (CENS) at http:// research.cens.ucla.edu.

5. At a recent exhibition, "Sense of the City," at the Canadian Centre for Architecture, the following citation from Anthony Synnott (1993) appeared in the opening room: "The reduction of the sensorium into five senses was first determined by Aristotle, but Galen said there were six, Erasmus Darwin thought there were twelve, and von Fray reduced them to eight. Zen

Buddhists say there is a sixth sense. Recent authorities calculate there are seventeen senses."

6. Île sans fil has installed over 50 hotspots providing service to 10,000 registered users in Montreal. A map of ISF hotspots is available at http://www.ilesansfil.org.

7. For more on this project, see http://www.spots-berlin.com/en/ index.php?col=2\&expo=.

\section{References}

Akyildiz, I.F., Su, W., Sankarasubramaniam, Y. and Cayirci E. 2002. "A Survey on Sensor Networks." IEEE Communications Magazine, August: 102-14.

Beigl, Michael. 2005. "Ubiquitous Computing: Computation Embedded in the World." In Georg Flachbart, and Peter Weibel (eds), Disappearing Architecture: From Real to Virtual to Quantum. Basel: Birkhauser.

Benjamin, Walter. 1968. "The Work of Art in the Age of Mechanical Reproduction." In Hanna Arendt (ed.), Illuminations. New York: HBJ, Schoken Books.

Buck-Morss, Susan. 1992. "Aesthetics and Anaesthetics: Walter Benjamin's Artwork Essay Reconsidered." October, 62: 3-41. Cavell, Richard. 2002. McLuhan in Space, Toronto: University of Toronto Press.

Connor, Steven. 2005. "The Menagerie of the Senses," presented at the sixth Synapsis conference, Bertinoro, Italy. Available online at http://www.bbk.ac.uk/english/skc/. Also, 2006. The Senses and Society, 1(1): 9-26.

Crary, Jonathan. 1990. Techniques of the Observer: On Vision and Modernity in the Nineteenth Century. Cambridge: MIT Press. Gabrys, Jennifer. n.d. "Telepathically Urban," in Cultures of 
Circulation, Will Straw and Alexandra Boutros (eds), Montreal: McGill-Queen's Press.

Forthcoming.

Hansen, Mark. 2000. Embodying Technesis: Technology Beyond Writing. Ann Arbor: University of Michigan Press.

Hayles, N. Katherine. 2005. "Computing the Human." Theory, Culture \& Society, 22(1): 131-51.

Howes, David (ed.). 2005. Empire of the Senses: The Sensual Culture Reader. Oxford: Berg.

McLuhan, Marshall. 1964. Understanding Media: The Extensions of Man. New York: McGrawHill.

Smith, Marquard and Joanne Morra (eds). 2005. The Prosthetic Impulse. Cambridge: MIT Press.

Synnott, Anthony. 1993. The Body Social. London, Routledge. 